\title{
Soluble guanylate cyclase stimulators increase sensitivity to cisplatin in head and neck squamous cell carcinoma cells
}

Traci R. Tuttle ${ }^{\mathrm{a}}$, Vinita Takiar ${ }^{\mathrm{b}}$, Bhavna Kumar ${ }^{\mathrm{c}}$, Pawan Kumar ${ }^{\mathrm{c}}$, Nira Ben-Jonathan ${ }^{\mathrm{a}}$

${ }^{a}$ Department of Cancer Biology, University of Cincinnati School of Medicine, Cincinnati, OH 45267, USA

${ }^{b}$ Department of Radiation Oncology, University of Cincinnati School of Medicine, Cincinnati, $\mathrm{OH} 45267, \mathrm{USA}$

${ }^{c}$ Department of Otolaryngology-Head and Neck Surgery, Comprehensive Cancer Center, The Ohio State University, Columbus, Ohio 43210, USA

*Corresponding author at: Department of Cancer Biology, The University of Cincinnati, 3125

Eden Ave, Cincinnati, OH 45267, USA

Key Words: cGMP, sGC, HNSCC, YC-1, BAY 41- 2272, apoptosis, EGFR, $\beta$-catenin 


\begin{abstract}
Head and neck squamous cell carcinoma (HNSCC) is an aggressive and often fatal disease. Cisplatin is the most common chemotherapeutic drug in the treatment of HNSCC, but intrinsic and acquired resistance are frequent, and severe side effects occur at high doses. The second messenger cyclic GMP (cGMP) is produced by soluble guanylate cyclase (sGC). We previously reported that activation of the cGMP signaling cascade caused apoptosis in HNSCC cells, while others found that this pathway enhances cisplatin efficacy in some cell types. Here we found that sGC stimulators reduced HNSCC cell viability synergistically with cisplatin, and enhanced apoptosis by cisplatin. Moreover, the sGC stimulators effectively reduced viability in cells with acquired cisplatin resistance, and were synergistic with cisplatin. The sGC stimulator BAY 412272 reduced expression of the pro-survival EGFR and $\beta$-catenin, and increased pro-apoptotic Bax, suggesting a potential mechanism for the anti-tumorigenic effects of these drugs. The sGC stimulator Riociguat is FDA-approved to treat pulmonary hypertension, and others are being studied for therapeutic use in several diseases. These drugs could provide valuable additions or alternatives to cisplatin in the treatment of HNSCC.
\end{abstract}




\section{Introduction}

Head and neck cancers (HNC) are diverse tumors within the oral cavity, oropharynx, hypopharynx and larynx [1-3]. Over 500,000 patients are diagnosed annually worldwide, with squamous cell carcinoma (HNSCC) representing $90 \%$ of the cases. Long term survival varies from $10-50 \%$, depending on tumor size, stage and resectability, and is less than 10 months for recurrent diseases. Tobacco use and alcohol abuse are major risk factors especially when combined. In addition, infection with human papillomavirus (HPV) is present in $\sim 25 \%$ of cases $[3 ; 4]$. Surgery and radiotherapy are the mainstay treatments for small tumors, while combined radiotherapy and chemotherapy are employed in advanced disease. Many tumors overexpress epidermal growth factor receptor (EGFR), and are treated with various EGFR antagonists [5;6]. In spite of recent advances, prognosis for recurrent/metastatic HNSCC remains dismal. Furthermore, disease progression or treatment can severely reduce the quality of life by causing facial disfiguration and impeding swallowing and speech [7].

The efficacy of chemotherapy is often compromised by intrinsic or acquired resistance [8]. Indeed, resistance to cisplatin, the most common chemotherapeutic drug in the treatment of HNC, necessitates the use of increased therapeutic doses, resulting in escalation of adverse side effects. There is, therefore, an urgent need for novel therapeutics in the treatment of HNSCC, which also can overcome resistance to cisplatin.

The cyclic GMP (cGMP)/Protein Kinase G (PKG) pathway serves as an intrinsic apoptotic pathway in glioma, breast and colon cancers [9-14]. Cyclic GMP is generated from GTP by two distinct guanylate cyclases (GC): particulate, which are membrane receptors for natriuretic peptides [15], and soluble (sGC), which are cytosolic receptors for nitric oxide (NO) [16]. Once produced, cGMP can be rapidly hydrolyzed to inactive 5'-GMP by cyclic nucleotide 
phosphodiesterases (PDEs), such as PDE5 [17]. The main downstream effectors of cGMP are two serine-threonine kinases, PKG-I and PKG-II [18]. Several drugs which target the cGMP/PKG pathway are approved for the treatment of nonmalignant conditions. For example, Riociguat, a NO-independent sGC stimulator, is used to treat pulmonary hypertension [19], while selective PDE5 inhibitors such as Tadalafil (Cialis) and Sildenafil (Viagra) are used to treat erectile dysfunction [20].

We recently reported that activation of the cGMP pathway in HNSCC cells by sGC activators or PDE5 inhibitors reduced cell viability and clonogenic growth and induced apoptosis [21]. Others found that YC-1, a sGC activator, as well as PDE5 inhibitors, enhance the efficacy of a variety of chemotherapeutic agents in certain cancers [22-24]. In addition, activation of the cGMP/PKG pathway reduces signaling via the EGFR [25] and $\beta$-catenin [26], pathways, both of which have been associated with HNSCC cell survival and chemoresistance [27;28].

The objectives of this study were to: 1) determine whether sGC activators enhance the efficacy of cisplatin to reduce cell viability and increase apoptosis in HNSCC cells, 2) examine the effects of sGC activators on cisplatin resistant cells, and 3) explore the effects of these drugs on signaling and pathways such as EGFR and $\beta$-catenin, and on apoptotic mediators such as Bax and Bcl-2.

\section{Materials and Methods}

\subsection{Cell lines and culture}

UMSCC-1 (UM1; oral cavity, HPV') and UMSCC-47 (UM47; tongue, $\mathrm{HPV}^{+}$) cells were purchased from the University of Michigan. CAL27 (tongue, HPV') parental cells and their cisplatin-resistant line (CAL27-CisR) were from Pawan Kumar at Ohio State University. Cells 
were maintained in DMEM containing 10\% FBS (Atlanta Biologicals, Flowery Branch, GA) and $50 \mu \mathrm{g} / \mathrm{ml}$ normocin (Invivogen, San Diego, CA). UM1 cells were cultured in increasing concentrations of cisplatin for 6 months to produce a cisplatin resistant subline UM1-CisR. CAL27-CisR and UM1-CisR cells were maintained in media containing $3 \mu \mathrm{M}$ cisplatin. For experiments, cells were starved for $24 \mathrm{~h}$ and then incubated with various treatments in DMEM containing 2\% charcoal/dextran-stripped FBS (Atlanta Biologicals) unless otherwise noted.

\subsection{Cell viability}

Cells were plated at 3000 cells/well in 96-well plates and treated for $72 \mathrm{~h}$ with the sGC stimulators BAY 41-2272 (BAY), YC-1 (Cayman Chemical, Ann Arbor, MI), or cisplatin (Sigma Aldrich, St. Louis, MO), or with a combination of cisplatin and the sGC stimulators. Cell viability was determined by the MTT assay (Affymetrix, Cleveland, OH). For drug combination studies, synergism or additivity were assessed by calculating the combination index (CI), according to Chou/Talalay [29], wherein synergism is defined as $\mathrm{CI}<1$, antagonism as $\mathrm{CI}>1$, and additive effects as $\mathrm{CI}=1$. The $\mathrm{CI}$ values were calculated using CompuSyn software (ComboSyn, Inc., Paramus, NJ).

\subsection{Flow Cytometry}

Cells plated at 300,000 cells/well in 6-well plates were treated for $48 \mathrm{~h}$ with the indicated drugs, and apoptosis was determined using FITC/Annexin V Apoptosis Detection Kit (BD Biosciences, San Jose, CA). Briefly, cells were incubated with FITC-Annexin V and propidium iodide for 15 min and then analyzed by flow cytometry, using a Cell Lab Quanta SC Flow Cytometer with accompanying software (Beckman Coulter). About 10,000 gated events were collected per treatment. 


\subsection{Real-time PCR}

Total RNA was isolated using the illustra RNAspin Mini kit from GE Healthcare (Buckinghamshire, UK), and was reverse transcribed using the $\mathrm{RT}^{2}$ First Strand cDNA kit from Qiagen (Hilden, Germany). Real-time PCR was performed on 30 ng of cDNA using Absolute Blue QPCR SYBR Green ROX mix (Thermo Scientific) on an Applied Biosystems StepOnePlus real-time PCR system. Changes in EGFR and CTNNB1 ( $\beta$-catenin) gene expression were calculated from the cycle threshold, after correcting for cDNA amounts using B2M ( $\beta$-2microglobulin) expression, according to Pfaffl et al [30]. The primer pairs were as follows: EGFR (F: CTAAGATCCCGTCCATCGCC, R: GGAGCCCAGCACTTTGATCT), CTNNBI (F: ATGACTCGAGCTCAGAGGGT, R: ATtGCACGTGTGGCAAGTTC), $\quad$ B2M $\quad$ (F: GGCATTCCTGAAGCTGAC, R: GAATCTTTGGAGTACGCTGG)

\subsection{Western blotting}

Cells were treated for $24 \mathrm{~h}$ and then lysed on ice in RIPA buffer containing protease inhibitors (Sigma Aldrich). Protein concentrations were determined by a BCA protein assay (Thermo Fisher), and $30 \mu \mathrm{g}$ were resolved on $12 \%$ SDS-PAGE, and transferred to PVDF membranes. After blocking with $0.5 \%$ BSA, membranes were incubated in primary antibodies against EGFR (cat\# 4267), $\beta$-catenin (cat\# 9582), Bax (cat\# 2772) or Bcl-2 (cat\# 2872) (Cell Signaling Technologies, Danvers, MA) for 24 h, followed by several washes and incubation with HRPconjugated secondary antibody (GE Healthcare). Blots were developed using SuperSignal West Pico chemiluminescence substrate (Thermo Fisher). The membranes were re-probed with $\beta$-actin antibody (Sigma Aldrich) as loading control. 


\subsection{Statistics}

Student's t-test or ANOVA with Tukey's post-hoc test were used where appropriate. P-values $\leq$ 0.05 were considered significant. All experiments were repeated at 2-3 times, unless otherwise noted.

\section{Results}

\subsection{BAY 41-2272 and YC-1 synergize with cisplatin to reduce HNSCC cell viability}

We first examined the effects of the sGC stimulators YC-1 and BAY 41-2272 (BAY) on the viability of HNSCC cells alone or in combination with cisplatin. CAL27, UM1 and UM47 cells were treated for $72 \mathrm{~h}$ with BAY, YC-1, or cisplatin alone, or with a combination of cisplatin with BAY or YC-1. As determined by the MTT assay, YC-1, BAY or cisplatin alone dosedependently reduced the viability of all three cell lines (Fig. 1). The combination of YC-1 or BAY with cisplatin synergistically reduced cell viability (Supplemental Table 1), as assessed by CI values $<1$, which designate a synergistic effect of the drug combination. BAY was more effective than YC-1 at lower doses, both alone and in combination with cisplatin.

\subsection{BAY 41-2272 and YC-1 increase the apoptotic effects of cisplatin}

We have previously shown that BAY reduced the viability of HNSCC cells primarily by inducing apoptosis, while only slightly reducing cell proliferation [21]. Here we examined whether YC-1 or BAY enhanced the apoptotic effects of cisplatin in HNSCC cells. CAL27 and UM1 cells were treated with YC-1, BAY or cisplatin alone, or with a combination of cisplatin with YC-1 or BAY for 48 hours. As determined by Annexin V staining and flow cytometric analysis, the combination of YC-1 with cisplatin increased apoptosis $\sim 70 \%$ over cisplatin alone 
in CAL27 cells, while BAY with cisplatin increased the apoptotic effect by $\sim 2$ fold over either drug alone (Fig. 2A). Similar results were obtained with UM1 cells (Fig 2B).

\subsection{The sGC stimulators are effective at reducing the viability of cisplatin-resistant cells}

We next examined the effects of sGC stimulators on the viability of HNSCC cells with acquired cisplatin resistance. The CAL27-CisR cells were previously generated by growing the cells in increasing doses of cisplatin over several weeks [31], while UM1-CisR cells were recently generated using the same approach. As compared to the parental cells, both cisplatin-resistant cells showed a significantly lower responsiveness to increasing doses of cisplatin (Fig 3A). In contrast, both CisR cells responded similarly to the parental cells when treated with YC-1 (Fig. 3B) or BAY (Fig. 3C), especially at the higher doses of the sGC stimulators.

\subsection{BAY 41-2272 synergizes with cisplatin to decrease the viability of cisplatin-resistant cells}

Next, CAL27-CisR and UM1-CisR cells were treated with combinations of cisplatin plus YC-1 or cisplatin plus BAY. The doses of YC-1 and BAY used here were the same or lower than those used in combination treatments with the parental cell lines, while the cisplatin doses were higher. As determined by the MTT assay, YC-1 in combination with cisplatin produced an additive effect on cell viability, while BAY in combination with cisplatin was synergistic (Fig 4 and Supplemental Table 2).

\subsection{BAY 41-2272 suppresses the expression of EGFR and $\beta$-catenin}

Previous reports have shown that drugs which increase cGMP and/or activate PKG reduce the levels of pro-survival proteins EGFR and $\beta$-catenin in some cancer cell types. We analyzed the protein levels of EGFR and $\beta$-catenin in CAL27 and UM47 cells treated with increasing doses of BAY. As determined by Western blotting, the levels of both proteins decreased dose- 
dependently in both cell lines (Fig. 5A and 5C). Analysis by real-time PCR revealed suppression of $E G F R$ and $C T N N B 1$ ( $\beta$-catenin) gene expression by $5 \mu \mathrm{M}$ BAY (Fig. 5B and 5D).

\subsection{BAY 41-2272 alters the expression of survival proteins}

We also examined changes in the pro-apoptotic protein Bax and anti-apoptotic Bcl-2 in response to increasing doses of BAY in CAL27 and UM47 cells. Fig. 6, top panel shows BAY-induced increase in Bax protein levels, while Bcl-2 remained relatively unchanged. Following densitometry, the change in the ratio of Bax to Bcl-2 was determined (Fig.6 lower panel). The ratio of Bax to Bcl-2 increased dose- dependently with BAY treatment in both cell lines.

\section{Discussion}

We are reporting that the sGC activators YC-1 and BAY 41-2272 (BAY) increase the efficacy of cisplatin to reduce viability and enhance apoptosis in HNSCC cells. Both drugs were also effective in cells with acquired cisplatin resistance (CisR). In addition, the sGC activators suppressed EGFR and $\beta$-catenin expression while increasing the levels of the pro-apoptotic protein Bax, suggesting potential mechanisms for the apoptotic and chemo-sensitizing effects of these drugs.

Previous studies have produced conflicting results on the consequences of activating the cGMP signaling on cancer cell survival and sensitivity to cisplatin. For example, NO donors and cGMP analogues reversed cisplatin resistance in fibrosarcoma cells [32], and PDE5 inhibitors synergized with cisplatin to reduce viability of medulloblastoma [22] and bladder cancer cells [33]. Conversely, sGC inhibitors enhanced the apoptotic effect of cisplatin in non-small cell lung

cancer cells [34]. A plausible explanation for this discrepancy is the differential expression of PKG isozymes, known to have disparate effects on cancer cell survival. Thus, PKG-I $\alpha$ has been 
reported to have pro-tumorigenic effects [34;35], while PKG-I $\beta$ [36;37] and PKG-II [13] had antineoplastic effects. Our previous studies found that HNSCC cell lines, including those used in the current study, express PKGI $\beta$ and PKGII, but had no detectable levels of PKGI $\alpha$ transcripts [21].

Although cisplatin is a first line treatment for advanced HNSCC, intrinsic and acquired resistance present a significant hindrance to treatment efficacy [38-40]. Decreased sensitivity to cisplatin necessitates dose escalation, resulting in significant side effects, including nephrotoxicity, ototoxicity, and peripheral nerve damage [41]. In the present study we clearly demonstrate that SGC stimulators are effective at reducing the viability of HNSCC cells with acquired cisplatin resistance, and furthermore, synergize with cisplatin in these cells. Such synergistic effects were evident in diverse HNSCC cell lines, derived from different anatomical locations within the oral cavity, and with variable HPV expression. Collectively, these data suggest that sGC stimulators could be highly beneficial as additions to cisplatin treatment for HNSCC, enabling the use of lower doses of cisplatin and minimizing side effects.

Treatment with relatively low doses of BAY reduced gene expression and protein levels of the pro-survival factors EGFR and $\beta$-catenin. EGFR is overexpressed in about $90 \%$ of HNSCC cases, and correlates with worse prognosis [42]. Degradation of EGFR has been associated with increased efficacy of cisplatin in head and neck cancer, and failure of responsiveness to cisplatin has been linked to reduced EGFR degradation [27]. In human non-small cell lung cancer xenografts, an EGFR monoclonal antibody enhanced the antitumor effects of cisplatin [43].

A previous study reported that $\beta$-catenin is transcriptionally upregulated in HNSCC cells with acquired cisplatin resistance [40]. In other studies, knock down of $\beta$-catenin expression sensitized ovarian [44] and lung [45] cancer cells to cisplatin. Moreover, overexpression of 
PKGI $\beta$ in colon cancer cells reduced $\beta$-catenin gene expression and signaling [26]. Inhibitors of PDE5, which presumably enhance the cGMP/PKG pathway, have also been found to exert proapoptotic effects in breast [46] and colon [47] cancer cells via inhibition of $\beta$-catenin expression and signaling downstream of PKG.

Treatment with BAY also lead to an increase expression of the pro-apoptotic protein Bax, while the pro-survival protein Bcl-2 remained unchanged. In response to apoptotic stimuli, Bax heterodimerizes with $\mathrm{Bcl}-2$ to suppress its pro-survival effects. As the ratio of Bax to Bcl-2 increases, cells are more likely to undergo apoptosis [48]. The Bax:Bcl-2 ratio positively correlated with the response to cisplatin and radiation treatment in bladder cancer [49].

In summary, we have shown that NO-independent stimulators of sGC enhance the effectiveness of cisplatin in both sensitive and resistant HNSCC cells. The mechanism underlying these effects may involve decreased expression of survival factors and increased amounts of proapoptotic factors. The sGC stimulator Riociguat (Adempas) is FDA-approved for chronic treatment of pulmonary hypertension, and has been well tolerated with minimal side effects. In the future, sGC stimulators could prove useful additions or alternatives to cisplatin treatment in patients with head and neck cancer.

\section{Acknowledgements}

This investigation was funded by NIH grants CA096613 and ES020909, DOD grants AR110050 and BC122992, and a pilot grant from Marlene Harris-Ride Cincinnati. We thank Drs William Barrett, the Department of Radiation Oncology, and Keith Casper, the Department of Otolaryngology, for providing financial support for these studies.

\section{Conflict of Interest}


The authors have nothing to declare.

\section{Reference List}

[1] J. A. de Souza, L. G. de Lima, E. E. Cohen, Pharmacoeconomic issues in head and neck oncology, Curr. Opin. Oncol. 25 (2013) 213-217.

[2] R. I. Haddad,D. M. Shin, Recent advances in head and neck cancer, N. Engl. J. Med. 359 (2008) 1143-1154.

[3] N. Denaro, E. G. Russi, V. Adamo, M. C. Merlano, State-of-the-art and emerging treatment options in the management of head and neck cancer: news from 2013, Oncology 86 (2014) 212-229.

[4] A. Psyrri,D. DiMaio, Human papillomavirus in cervical and head-and-neck cancer, Nat. Clin. Pract. Oncol. 5 (2008) 24-31.

[5] K. Dorsey,M. Agulnik, Promising new molecular targeted therapies in head and neck cancer, Drugs 73 (2013) 315-325.

[6] B. Burtness, J. E. Bauman, T. Galloway, Novel targets in HPV-negative head and neck cancer: overcoming resistance to EGFR inhibition, Lancet Oncol. 14 (2013) e302-e309.

[7] C. J. Wang,R. Knecht, Current concepts of organ preservation in head and neck cancer, Eur. Arch. Otorhinolaryngol. 268 (2011) 481-487.

[8] C. Holohan, S. S. Van, D. B. Longley, P. G. Johnston, Cancer drug resistance: an evolving paradigm, Nat. Rev. Cancer 13 (2013) 714-726.

[9] D. D. Browning, I. K. Kwon, R. Wang, cGMP-dependent protein kinases as potential targets for colon cancer prevention and treatment, Future. Med. Chem. 2 (2010) 65-80.

[10] F. Fallahian, F. Karami-Tehrani, S. Salami, M. Aghaei, Cyclic GMP induced apoptosis via protein kinase $\mathrm{G}$ in oestrogen receptor-positive and -negative breast cancer cell lines, FEBS J. 278 (2011) 3360-3369. 
[11] A. M. Fajardo, G. A. Piazza, H. N. Tinsley, The role of cyclic nucleotide signaling pathways in cancer: targets for prevention and treatment, Cancers. (Basel) 6 (2014) 436458.

[12] K. Mujoo, V. G. Sharin, E. Martin, B. K. Choi, C. Sloan, L. E. Nikonoff, A. Y. Kots, F. Murad, Role of soluble guanylyl cyclase-cyclic GMP signaling in tumor cell proliferation, Nitric. Oxide. 22 (2010) 43-50.

[13] F. J. Swartling, M. Ferletta, M. Kastemar, W. A. Weiss, B. Westermark, Cyclic GMPdependent protein kinase II inhibits cell proliferation, Sox9 expression and Akt phosphorylation in human glioma cell lines, Oncogene 28 (2009) 3121-3131.

[14] H. C. Wen, C. P. Chuu, C. Y. Chen, S. G. Shiah, H. J. Kung, K. L. King, L. C. Su, S. C. Chang, C. H. Chang, Elevation of soluble guanylate cyclase suppresses proliferation and survival of human breast cancer cells, PLoS. One. 10 (2015) e0125518.

[15] R. K. Sharma,T. Duda, Membrane guanylate cyclase, a multimodal transduction machine: history, present, and future directions, Front Mol. Neurosci. 7 (2014) 56.

[16] O. Gileadi, Structures of soluble guanylate cyclase: implications for regulatory mechanisms and drug development, Biochem. Soc. Trans. 42 (2014) 108-113.

[17] M. F. Azevedo, F. R. Faucz, E. Bimpaki, A. Horvath, I. Levy, R. B. de Alexandre, F. Ahmad, V. Manganiello, C. A. Stratakis, Clinical and molecular genetics of the phosphodiesterases (PDEs), Endocr. Rev. 35 (2014) 195-233.

[18] S. H. Francis,J. D. Corbin, Cyclic nucleotide-dependent protein kinases: intracellular receptors for cAMP and cGMP action, Crit Rev. Clin. Lab Sci. 36 (1999) 275-328.

[19] J. E. Cannon,J. Pepke-Zaba, Riociguat for pulmonary hypertension, Expert Rev. Clin. Pharmacol. 7 (2014) 259-270.

[20] P. J. Wright, Comparison of phosphodiesterase type 5 (PDE5) inhibitors, Int. J. Clin. Pract. 60 (2006) 967-975.

[21] T. R. Tuttle, M. L. Mierzwa, S. I. Wells, S. R. Fox, N. Ben-Jonathan, The cyclic GMP/protein kinase G pathway as a therapeutic target in head and neck squamous cell carcinoma, Cancer Lett. 370 (2016) 279-285.

[22] J. L. Roberts, L. Booth, A. Conley, N. Cruickshanks, M. Malkin, R. C. Kukreja, S. Grant, A. Poklepovic, P. Dent, PDE5 inhibitors enhance the lethality of standard of care chemotherapy in pediatric CNS tumor cells, Cancer Biol. Ther. 15 (2014) 758-767.

[23] C. S. Lee, S. W. Kwak, Y. J. Kim, S. A. Lee, E. S. Park, S. C. Myung, W. Kim, M. S. Lee, J. J. Lee, Guanylate cyclase activator YC-1 potentiates apoptotic effect of licochalcone A on human epithelial ovarian carcinoma cells via activation of death receptor and mitochondrial pathways, Eur. J. Pharmacol. 683 (2012) 54-62. 
[24] C. K. Lau, Z. F. Yang, S. P. Lam, C. T. Lam, P. Ngai, K. H. Tam, R. T. Poon, S. T. Fan, Inhibition of Stat3 activity by $\mathrm{YC}-1$ enhances chemo-sensitivity in hepatocellular carcinoma, Cancer Biol. Ther. 6 (2007) 1900-1907.

[25] L. Jiang, Y. Chen, Y. Li, T. Lan, M. Wu, Y. Wang, H. Qian, Type II cGMP-dependent protein kinase inhibits ligandinduced activation of EGFR in gastric cancer cells, Mol. Med. Rep. 9 (2014) 1405-1409.

[26] I. K. Kwon, R. Wang, M. Thangaraju, H. Shuang, K. Liu, R. Dashwood, N. Dulin, V. Ganapathy, D. D. Browning, PKG inhibits TCF signaling in colon cancer cells by blocking beta-catenin expression and activating FOXO4, Oncogene 29 (2010) 34233434.

[27] A. Ahsan, S. M. Hiniker, S. G. Ramanand, S. Nyati, A. Hegde, A. Helman, R. Menawat, M. S. Bhojani, T. S. Lawrence, M. K. Nyati, Role of epidermal growth factor receptor degradation in cisplatin-induced cytotoxicity in head and neck cancer, Cancer Res. 70 (2010) 2862-2869.

[28] F. Yang, Q. Zeng, G. Yu, S. Li, C. Y. Wang, Wnt/beta-catenin signaling inhibits death receptor-mediated apoptosis and promotes invasive growth of HNSCC, Cell Signal. 18 (2006) 679-687.

[29] T. C. Chou, Drug combination studies and their synergy quantification using the ChouTalalay method, Cancer Res. 70 (2010) 440-446.

[30] M. W. Pfaffl, G. W. Horgan, L. Dempfle, Relative expression software tool (REST) for group-wise comparison and statistical analysis of relative expression results in real-time PCR, Nucleic Acids Res. 30 (2002) e36.

[31] B. Kumar, A. Yadav, J. C. Lang, M. J. Cipolla, A. C. Schmitt, N. Arradaza, T. N. Teknos, P. Kumar, YM155 reverses cisplatin resistance in head and neck cancer by decreasing cytoplasmic survivin levels, Mol. Cancer Ther. 11 (2012) 1988-1998.

[32] H. Eichholtz-Wirth, Reversal of radiation-induced cisplatin resistance in murine fibrosarcoma cells by selective modulation of the cyclic GMP-dependent transduction pathway, Br. J. Cancer 72 (1995) 287-292.

[33] L. Booth, J. L. Roberts, N. Cruickshanks, A. Conley, D. E. Durrant, A. Das, P. B. Fisher, R. C. Kukreja, S. Grant, A. Poklepovic, P. Dent, Phosphodiesterase 5 inhibitors enhance chemotherapy killing in gastrointestinal/genitourinary cancer cells, Mol. Pharmacol. 85 (2014) 408-419.

[34] J. C. Wong, M. Bathina, R. R. Fiscus, Cyclic GMP/protein kinase G type-Ialpha (PKGIalpha) signaling pathway promotes CREB phosphorylation and maintains higher c-IAP1, livin, survivin, and Mcl-1 expression and the inhibition of PKG-Ialpha kinase activity synergizes with cisplatin in non-small cell lung cancer cells, J. Cell Biochem. 113 (2012) 3587-3598. 
[35] E. L. Leung, J. C. Wong, M. G. Johlfs, B. K. Tsang, R. R. Fiscus, Protein kinase G type Ialpha activity in human ovarian cancer cells significantly contributes to enhanced Src activation and DNA synthesis/cell proliferation, Mol. Cancer Res. 8 (2010) 578-591.

[36] F. Fallahian, F. Karami-Tehrani, S. Salami, Induction of apoptosis by type Ibeta protein kinase $\mathrm{G}$ in the human breast cancer cell lines MCF-7 and MDA-MB-468, Cell Biochem. Funct. 30 (2012) 183-190.

[37] Y. Hou, N. Gupta, P. Schoenlein, E. Wong, R. Martindale, V. Ganapathy, D. Browning, An anti-tumor role for cGMP-dependent protein kinase, Cancer Lett. 240 (2006) 60-68.

[38] L. Kelland, The resurgence of platinum-based cancer chemotherapy, Nat. Rev. Cancer 7 (2007) 573-584.

[39] J. A. Bauer, B. Kumar, K. G. Cordell, M. E. Prince, H. H. Tran, G. T. Wolf, D. B. Chepeha, T. N. Teknos, S. Wang, A. Eisbruch, C. I. Tsien, S. G. Urba, F. P. Worden, J. Lee, K. A. Griffith, J. M. Taylor, N. D'Silva, S. J. Wang, K. G. Wolter, B. Henson, S. G. Fisher, T. E. Carey, C. R. Bradford, Targeting apoptosis to overcome cisplatin resistance: a translational study in head and neck cancer, Int. J. Radiat. Oncol. Biol. Phys. 69 (2007) S106-S108.

[40] E. M. Gosepath, N. Eckstein, A. Hamacher, K. Servan, J. G. von, H. Lage, B. Gyorffy, H. D. Royer, M. U. Kassack, Acquired cisplatin resistance in the head-neck cancer cell line Cal27 is associated with decreased DKK1 expression and can partially be reversed by overexpression of DKK1, Int. J. Cancer 123 (2008) 2013-2019.

[41] L. Galluzzi, L. Senovilla, I. Vitale, J. Michels, I. Martins, O. Kepp, M. Castedo, G. Kroemer, Molecular mechanisms of cisplatin resistance, Oncogene 31 (2012) 18691883.

[42] A. R. Hansen,L. L. Siu, Epidermal growth factor receptor targeting in head and neck cancer: have we been just skimming the surface?, J. Clin. Oncol. 31 (2013) 1381-1383.

[43] T. Yoshida, I. Okamoto, T. Iwasa, M. Fukuoka, K. Nakagawa, The anti-EGFR monoclonal antibody blocks cisplatin-induced activation of EGFR signaling mediated by HB-EGF, FEBS Lett. 582 (2008) 4125-4130.

[44] H. Zhao, W. Wei, Y. Sun, J. Gao, Q. Wang, J. Zheng, Interference with the expression of beta-catenin reverses cisplatin resistance in A2780/DDP cells and inhibits the progression of ovarian cancer in mouse model, DNA Cell Biol. 34 (2015) 55-62.

[45] J. Zhang, J. Liu, H. Li, J. Wang, betacatenin signaling pathway regulates cisplatin resistance in lung adenocarcinoma cells by upregulating Bclxl, Mol. Med. Rep. 13 (2016) 2543-2551.

[46] H. N. Tinsley, B. D. Gary, A. B. Keeton, W. Lu, Y. Li, G. A. Piazza, Inhibition of PDE5 by sulindac sulfide selectively induces apoptosis and attenuates oncogenic Wnt/beta- 
catenin-mediated transcription in human breast tumor cells, Cancer Prev. Res. (Phila) 4 (2011) 1275-1284.

[47] N. Li, Y. Xi, H. N. Tinsley, E. Gurpinar, B. D. Gary, B. Zhu, Y. Li, X. Chen, A. B. Keeton, A. H. Abadi, M. P. Moyer, W. E. Grizzle, W. C. Chang, M. L. Clapper, G. A. Piazza, Sulindac selectively inhibits colon tumor cell growth by activating the cGMP/PKG pathway to suppress Wnt/beta-catenin signaling, Mol. Cancer Ther. 12 (2013) 1848-1859.

[48] Z. N. Oltvai, C. L. Milliman, S. J. Korsmeyer, Bcl-2 heterodimerizes in vivo with a conserved homolog, Bax, that accelerates programmed cell death, Cell 74 (1993) 609619.

[49] H. Matsumoto, T. Wada, K. Fukunaga, S. Yoshihiro, H. Matsuyama, K. Naito, Bax to Bcl-2 ratio and $\mathrm{Ki}-67$ index are useful predictors of neoadjuvant chemoradiation therapy in bladder cancer, Jpn. J. Clin. Oncol. 34 (2004) 124-130.

\section{Figure Legends}

Fig 1. The sGC stimulators synergize with cisplatin to reduce the viability of HNSCC cells. The HNSCC cell lines UMSCC-1 (UM1) (A), CAL27 (B) and UMSCC-47 (UM47) (C) were treated with increasing doses of YC-1 (left panels) or BAY (right panels) alone, or together with the indicated doses of cisplatin for $72 \mathrm{~h}$. Cell viability was determined by the MTT assay. Each value is a mean \pm SEM of 6 replicates.

Fig 2. The sGC stimulators enhance the apoptotic effect of cisplatin. UM1 (A) and CAL27 (B) cells were treated with YC-1 (left panels) or BAY (right panels) at the indicated doses, alone or together with cisplatin for $48 \mathrm{~h}$. Cells were stained with FITC-Annexin V and propidium iodide and were analyzed by flow cytometry. Each value is a mean \pm SEM of 3 replicates. * indicates significant $(\mathrm{p}<0.05)$ vs. control, \# indicates significant vs. YC-1 alone and \& indicates significant vs. cisplatin alone. 
Fig 3. Cisplatin resistant HNSCC cells are sensitive to BAY and YC-1. UM1-CisR and CAL27CisR cells and their parental lines were treated with increasing doses of cisplatin (A), YC-1 (B) or BAY (C) for 72h. Cell viability was determined by MTT assay. Each value is a mean \pm SEM of 6 replicates. * indicates significant $(\mathrm{p}<0.05)$ vs. control.

Fig 4. The sGC stimulators synergize with cisplatin to reduce the viability of cisplatin resistant HNSCC cells. UM1-CisR (A) and CAL27-CisR (B) were treated with increasing doses of YC-1 (left panels) or BAY (right panels) alone, or with the indicated doses of cisplatin for $72 \mathrm{~h}$. Cell viability was determined by MTT assay. Each value is a mean \pm SEM of 6 replicates.

Fig 5. Treatment with BAY reduces the expression of EGFR and $\beta$-catenin in CAL27 and UM47 cells. Cells were treated with increasing doses of BAY for 48 hours, lysed and analyzed for EGFR (A) or $\beta$-catenin (C) by Western blotting. Expression of EGFR (B) and CTNNB1 (D) mRNA was examined by real-time qPCR. Each value is a mean \pm SEM of 3 replicates. * indicates significant $(\mathrm{p}<0.05)$ vs. control.

Fig 6. BAY increases the ratio of pro-apoptotic Bax to anti-apoptotic Bcl-2 in CAL27 and UM47 cells. Cells were treated with the indicated doses of BAY for $48 \mathrm{~h}$, lysed and analyzed for Bax and Bcl-2 by Western blotting (upper panel); $\beta$-actin served as a loading control. Protein levels were quantified by densitometry, and the Bax:Bcl-2 ratio is shown in the lower panel. Each value is a mean \pm SEM of 3 replicates. * indicates significant $(\mathrm{p}<0.05)$ vs. control. 


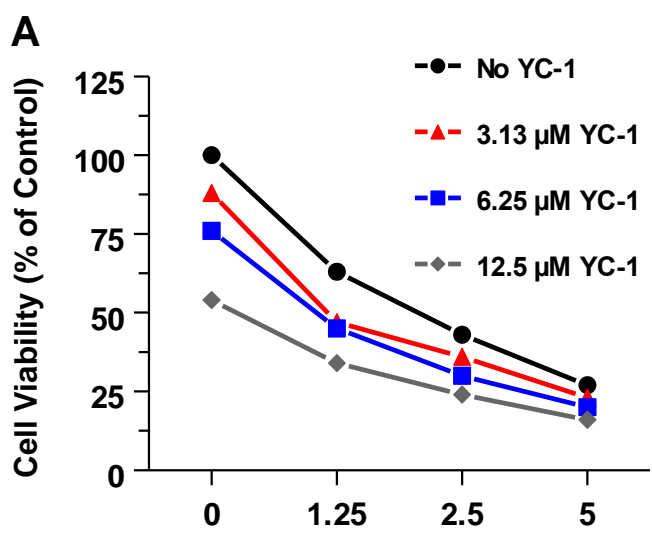

Cisplatin $(\mu \mathrm{M})$

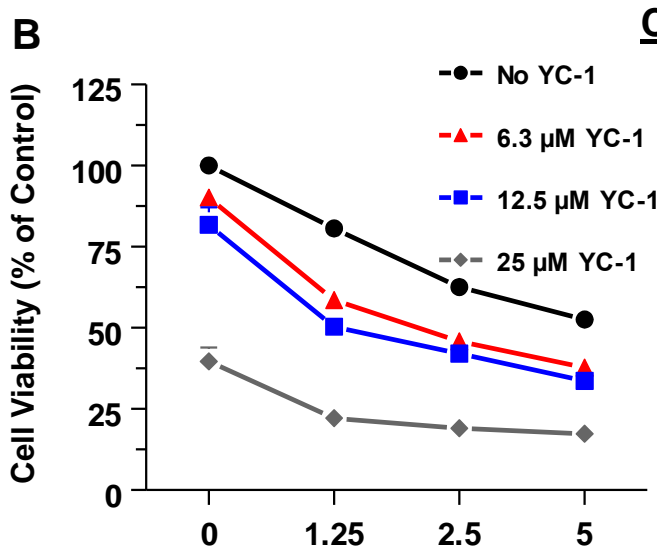

Cisplatin $(\mu \mathrm{M})$

C

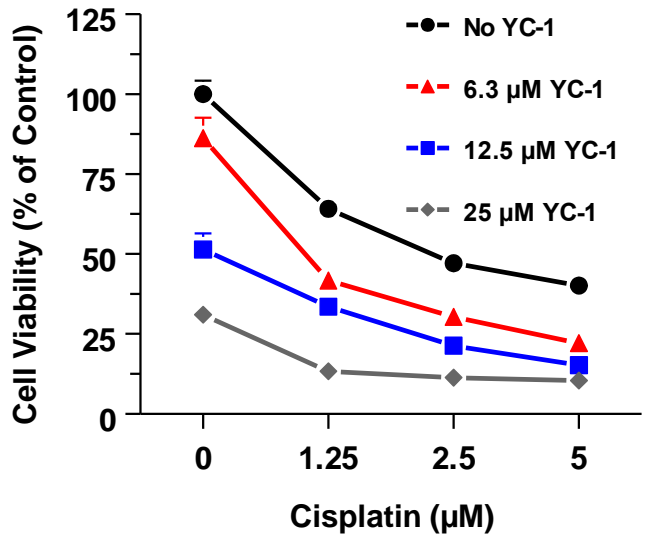

UM1

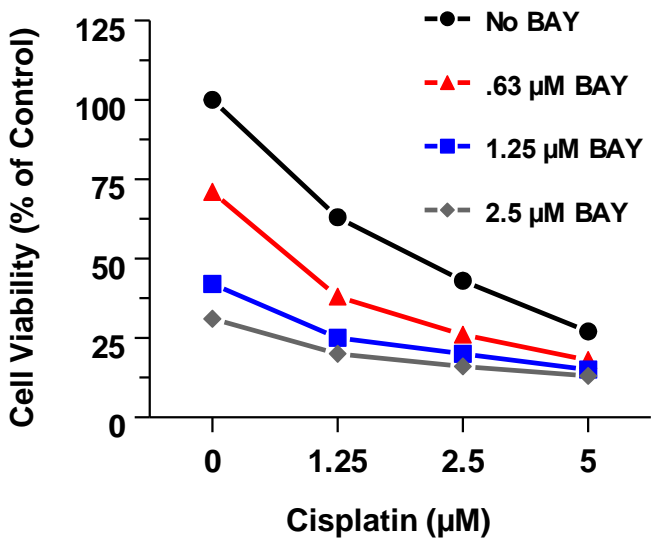

CAL27

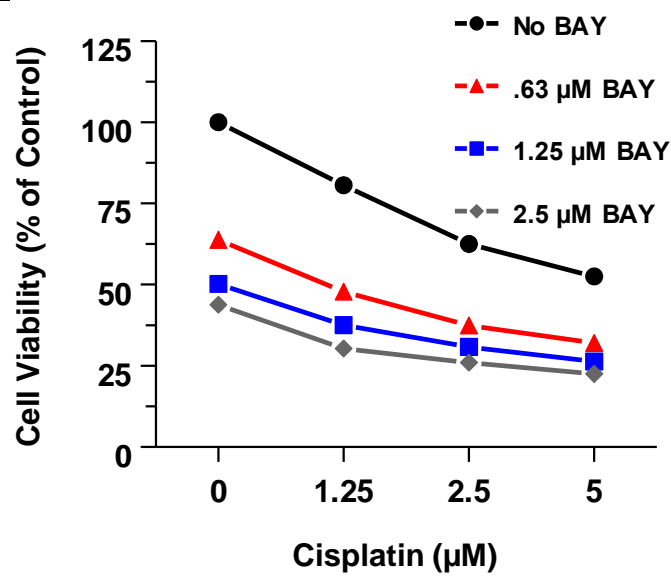

UM47

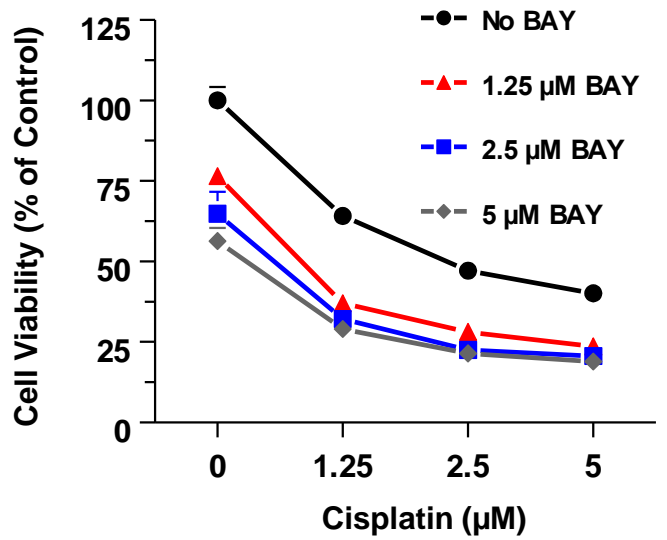



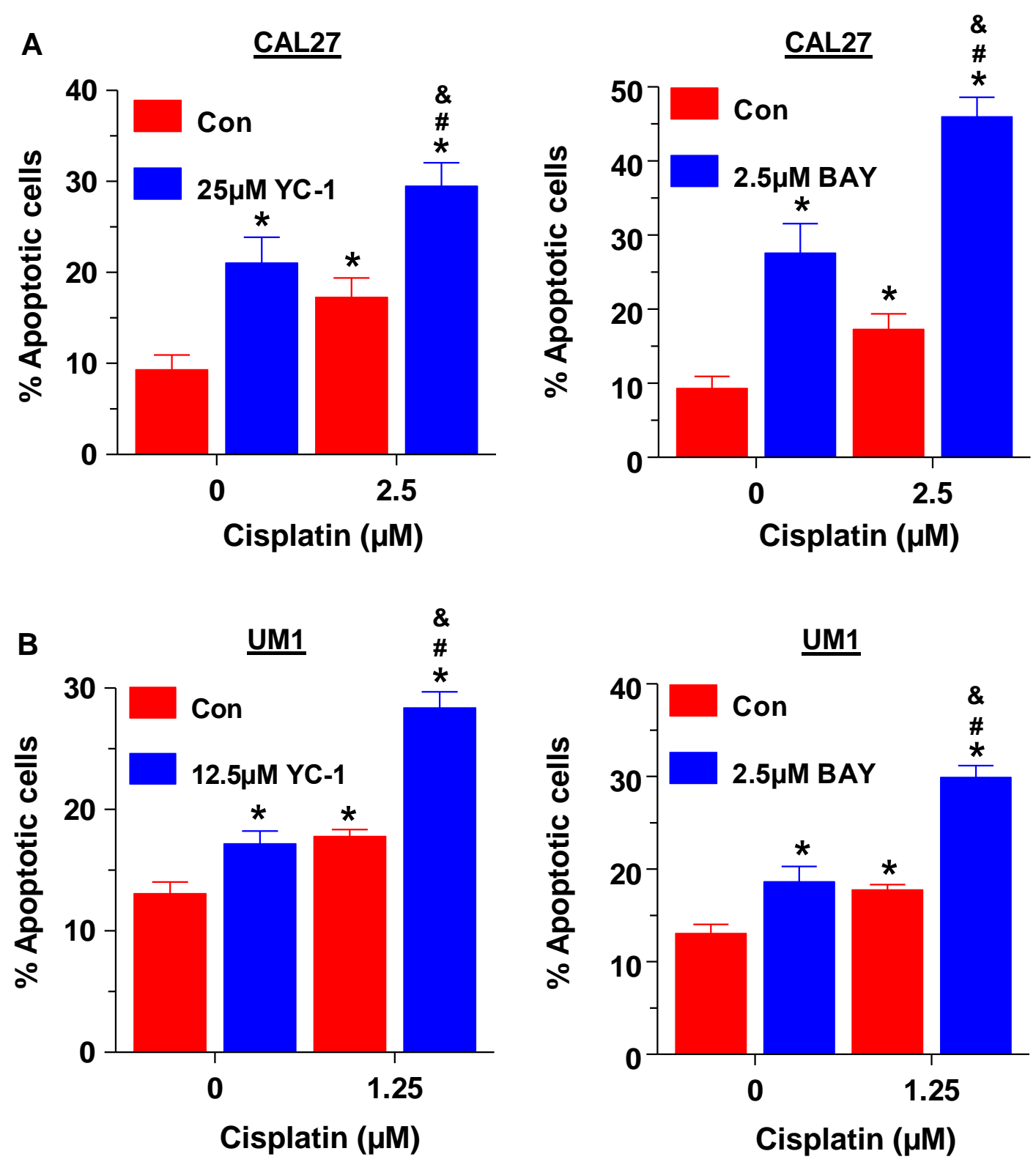

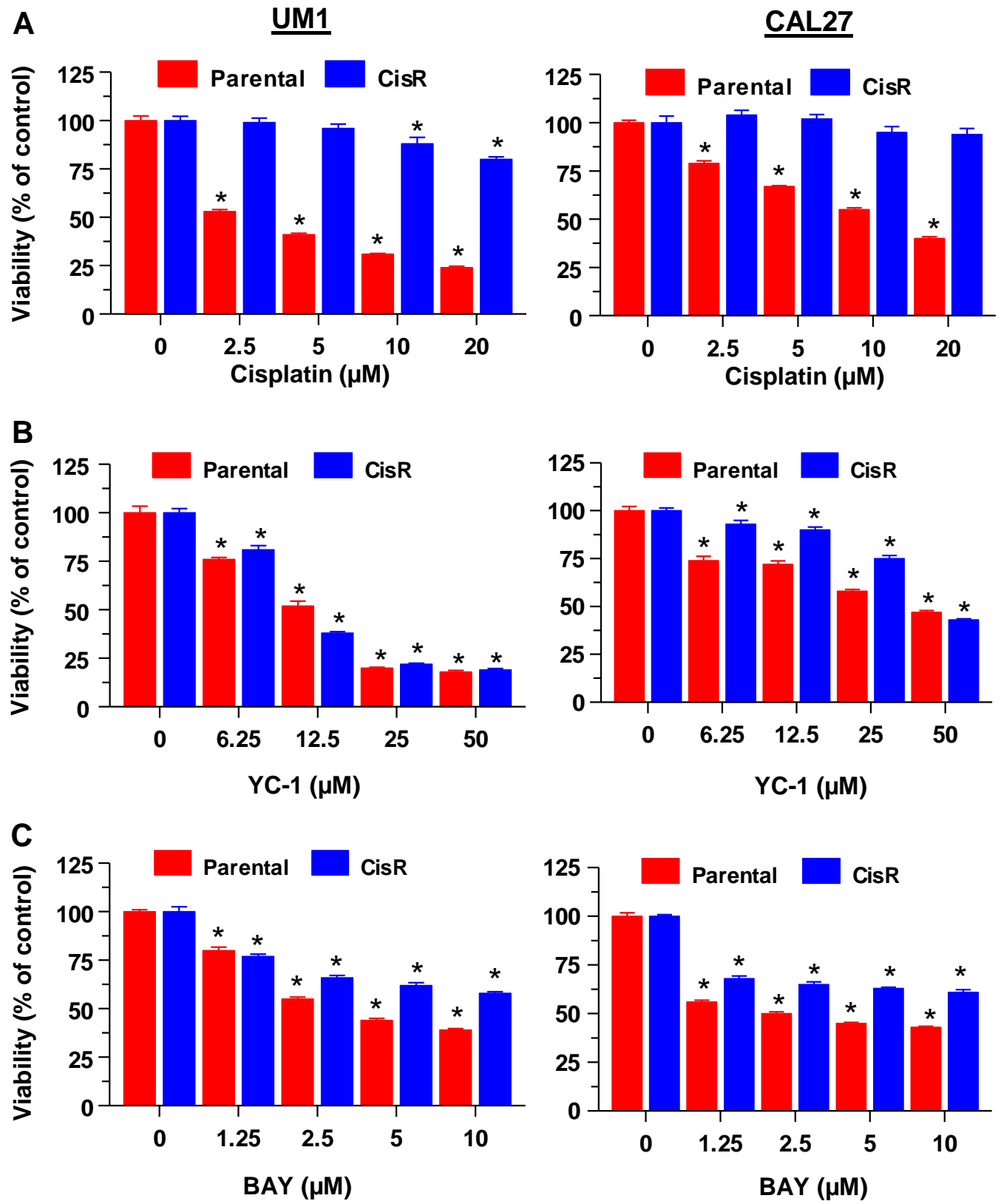


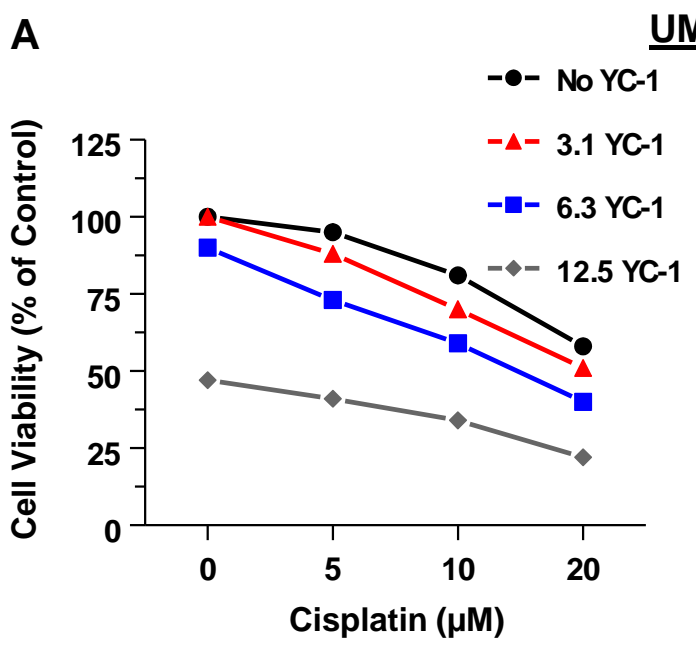

UM1 CisR

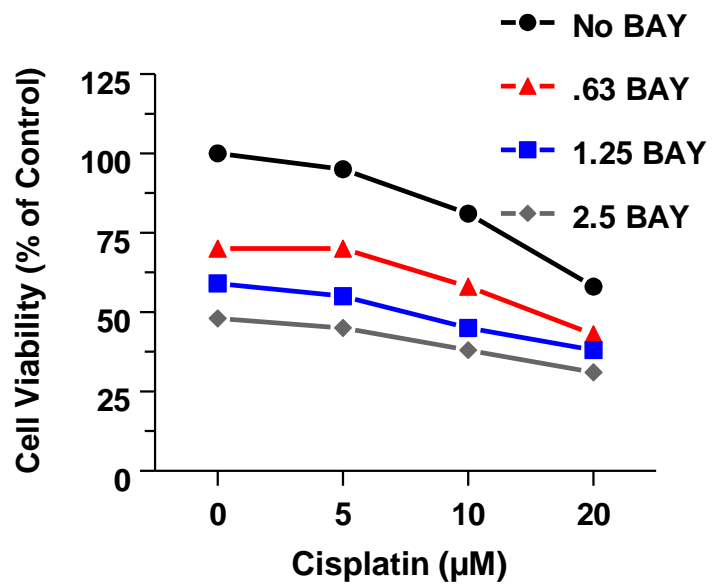

B

CAL27 CisR
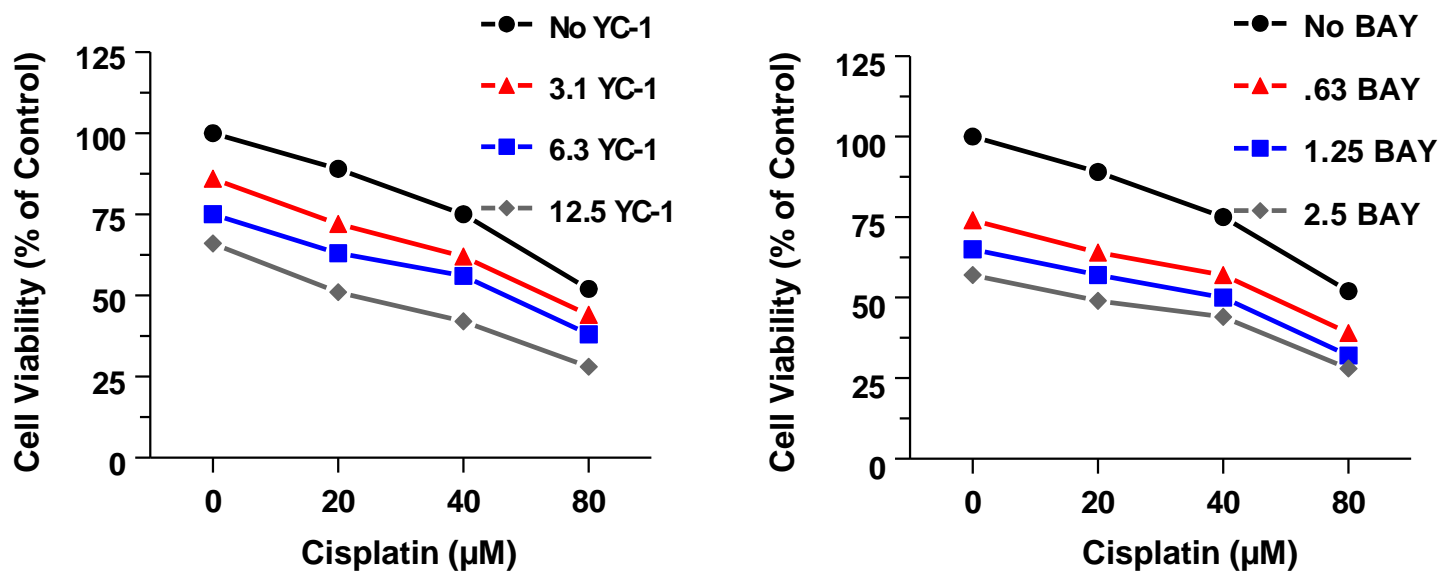
A

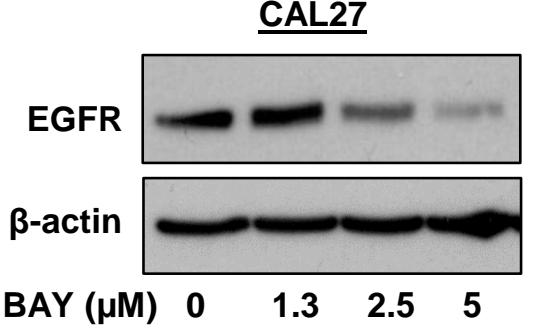

B

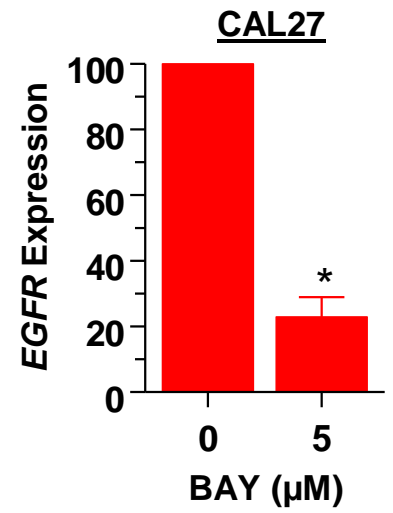

C
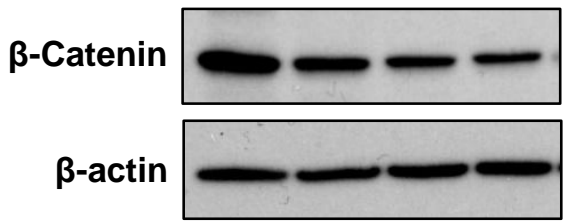

$\begin{array}{lllll}\operatorname{BAY}(\mu \mathrm{M}) & 0 & 1.3 & 2.5 & 5\end{array}$
UM47
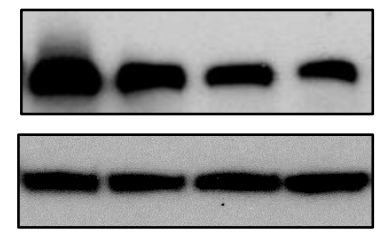

$\begin{array}{llll}0 & 1.3 & 2.5 & 5\end{array}$
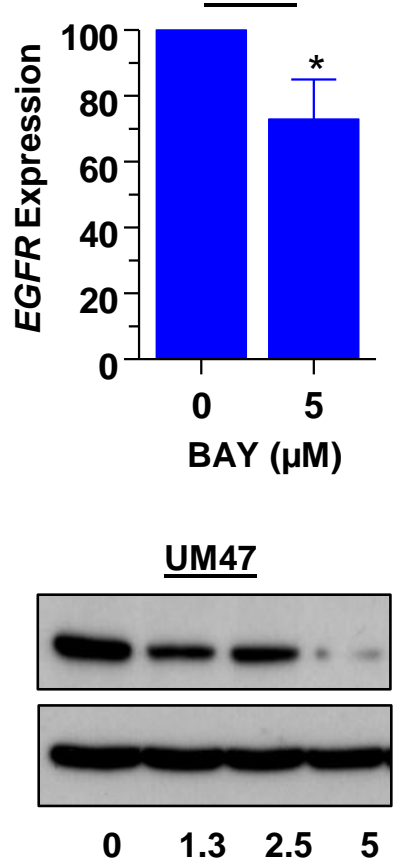

D

CAL27

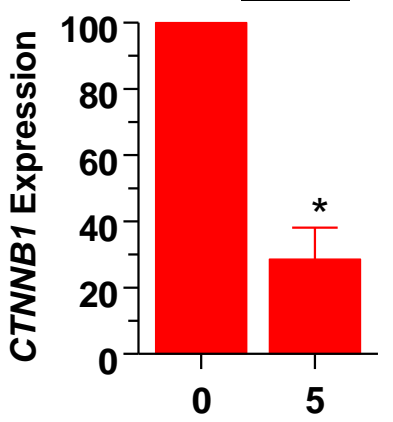

BAY $(\mu \mathrm{M})$

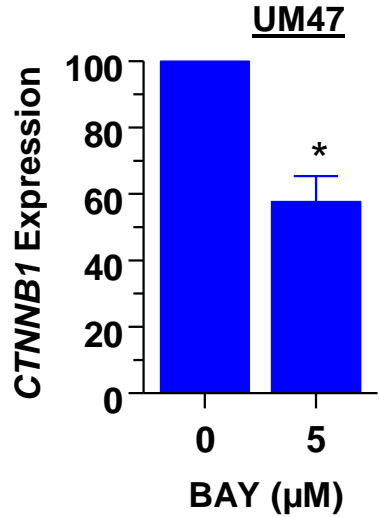



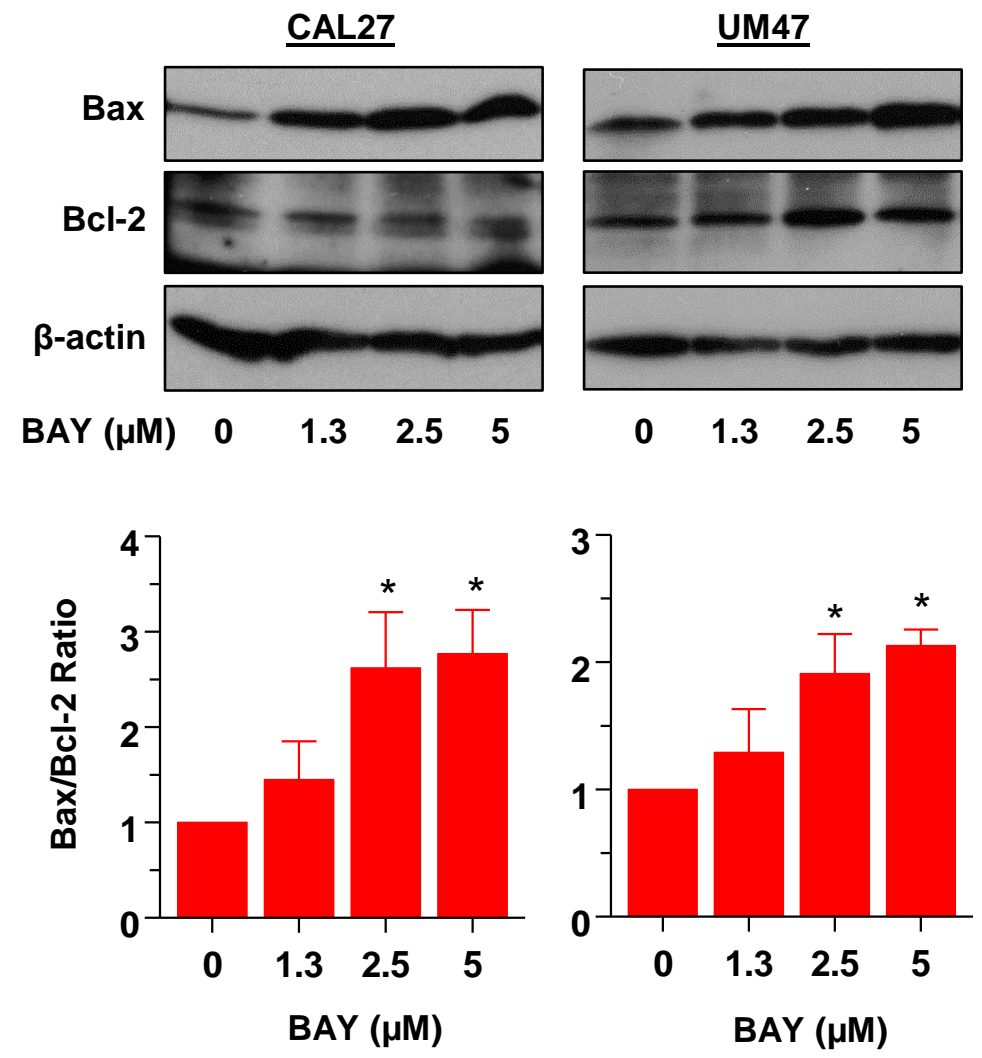

Figure 6 